\title{
Doping Graphene with Metal Contacts
}

\author{
G. Giovannetti, ${ }^{1,2}$ P. A. Khomyakov, ${ }^{2}$ G. Brocks, ${ }^{2}$ V. M. Karpan, ${ }^{2}$ J. van den Brink, ${ }^{1,3}$ and P. J. Kelly ${ }^{2}$ \\ ${ }^{1}$ Instituut-Lorentz for Theoretical Physics, Universiteit Leiden, P.O. Box 9506, 2300 RA Leiden, The Netherlands \\ ${ }^{2}$ Faculty of Science and Technology and MESA ${ }^{+}$Institute for Nanotechnology, University of Twente, \\ P.O. Box 217, 7500 AE Enschede, The Netherlands \\ ${ }^{3}$ Institute for Molecules and Materials, Radboud Universiteit, Nijmegen, The Netherlands
}

(Received 15 February 2008; published 10 July 2008)

\begin{abstract}
Making devices with graphene necessarily involves making contacts with metals. We use density functional theory to study how graphene is doped by adsorption on metal substrates and find that weak bonding on $\mathrm{Al}, \mathrm{Ag}, \mathrm{Cu}, \mathrm{Au}$, and $\mathrm{Pt}$, while preserving its unique electronic structure, can still shift the Fermi level with respect to the conical point by $\sim 0.5 \mathrm{eV}$. At equilibrium separations, the crossover from $p$-type to $n$-type doping occurs for a metal work function of $\sim 5.4 \mathrm{eV}$, a value much larger than the graphene work function of $4.5 \mathrm{eV}$. The numerical results for the Fermi level shift in graphene are described very well by a simple analytical model which characterizes the metal solely in terms of its work function, greatly extending their applicability.
\end{abstract}

DOI: 10.1103/PhysRevLett.101.026803

Recent progress in depositing a single graphene sheet on an insulating substrate by micromechanical cleavage enables electron transport experiments on this twodimensional system [1,2]. Such experiments demonstrate an exceptionally high electron mobility in graphene, quantization of the conductivity, and a zero-energy anomaly in the quantum Hall effect, in agreement with theoretical predictions [3-7]. The spectacular effects arise from graphene's unique electronic structure. Although it has a zero band gap and a vanishing density of states (DOS) at the Fermi energy, graphene exhibits metallic behavior due to topological singularities at the $\mathrm{K}$ points in the Brillouin zone $[3,4]$ where the conduction and valence bands touch in conical (Dirac) points and the dispersion is essentially linear within $\pm 1 \mathrm{eV}$ of the Fermi energy.

In a freestanding graphene layer the Fermi energy coincides with the conical points but adsorption on metallic (or insulating) substrates can alter its electronic properties significantly [8-15]. Since electronic transport measurements through a graphene sheet require contacts to metal electrodes $[2,12,16,17]$, it is essential to have a full understanding of the physics of metal-graphene interfaces. In this Letter we use first-principles calculations at the level of density functional theory (DFT) to study the adsorption of graphene on a series of metal substrates. The (111) surfaces of $\mathrm{Al}, \mathrm{Co}, \mathrm{Ni}, \mathrm{Cu}, \mathrm{Pd}, \mathrm{Ag}, \mathrm{Pt}$, and $\mathrm{Au}$, covering a wide range of work functions and chemical bonding, form a suitable system for a systematic study.

Our results show that these substrates can be divided into two classes. The characteristic electronic structure of graphene is significantly altered by chemisorption on $\mathrm{Co}, \mathrm{Ni}$, and $\mathrm{Pd}$ but is preserved by weak adsorption on $\mathrm{Al}, \mathrm{Cu}, \mathrm{Ag}$, $\mathrm{Au}$, and Pt. Even when the bonding is weak, however, the metal substrates cause the Fermi level to move away from the conical points in graphene, resulting in doping with either electrons or holes. The sign and amount of doping
PACS numbers: 73.63.-b, 73.20.Hb, 73.40.Ns, 81.05.Uw

can be deduced from the difference of the metal and graphene work functions only when they are so far apart that there is no wave function overlap. At the equilibrium separation, the doping level is strongly affected by an interface potential step arising from the direct metalgraphene interaction.

Based upon the DFT results, we develop a phenomenological model to describe the doping of graphene, taking into account the metal-graphene interaction. The model uses only the work functions of graphene and of the clean metal surfaces as input to predict the Fermi level shift in graphene with respect to the conical points, i.e., both the type and concentration of the charge carriers. The model also predicts how metal work functions are modified by adsorption of graphene.

Some details of how DFT ground state energies and optimized geometries are calculated for graphene on metal (111) surfaces are given in Ref. [18]. We fix the in-plane lattice constant of graphene to its optimized value $a=$ $2.445 \AA$ and adapt the lattice constants of the metals accordingly. The graphene honeycomb lattice then matches the triangular lattice of the metal (111) surfaces in the unit cells shown in Fig. 1. The approximation made by this procedure is reasonable, since the mismatch with the optimized metal lattice parameters is only $0.8 \%-3.8 \%$. We have verified explicitly that the structures shown in Fig. 1 represent the most stable configurations of graphene on the metal substrates studied. The equilibrium separations, binding energies, and work functions are listed in Table I.

The results immediately show that the metals can be divided into two classes. Graphene is chemisorbed on $\mathrm{Co}, \mathrm{Ni}$, and $\operatorname{Pd}(111)$, leading to binding energies $\Delta E \sim$ $0.1 \mathrm{eV}$ per carbon atom and equilibrium separations $d_{\text {eq }} \lesssim 2.3 \AA$. In contrast, adsorption on $\mathrm{Al}, \mathrm{Cu}, \mathrm{Ag}, \mathrm{Au}$, and $\mathrm{Pt}(111)$ leads to a weaker bonding, $\Delta E \lesssim$ $0.04 \mathrm{eV}$ per carbon atom, and larger equilibrium separa- 


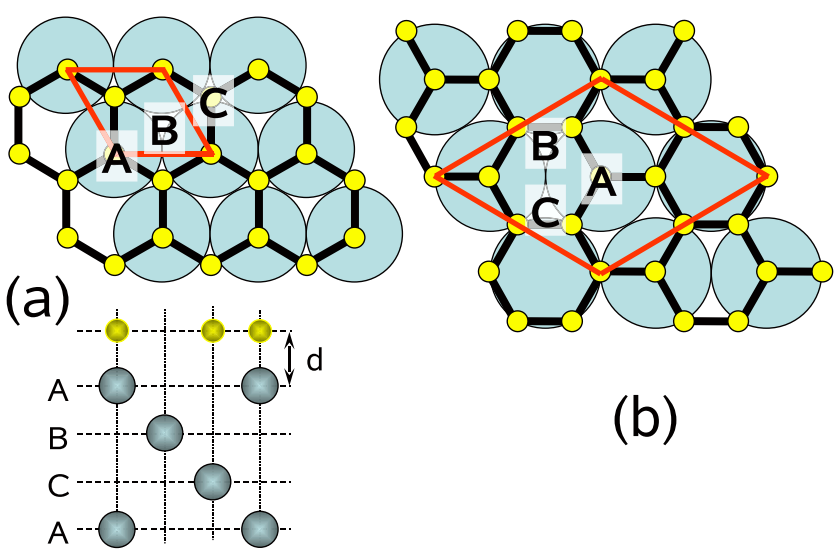

FIG. 1 (color online). The most stable configurations of graphene (a) on $\mathrm{Cu}, \mathrm{Ni}$, and $\mathrm{Co}$ (111) with one carbon atom on top of a metal atom (A site), and the second carbon on a hollow site (C site) and (b) on $\mathrm{Al}, \mathrm{Au}, \mathrm{Pd}$, and $\mathrm{Pt}(111)$ in a unit cell with 8 carbon atoms and 3 metal atoms per layer.

tions, $d_{\mathrm{eq}} \sim 3.3 \AA$. These results are in agreement with previous calculations and experimental data $[8,10,12,19,20]$.

To identify the changes in the graphene electronic structure induced by adsorption, we calculate the band structures as illustrated in Fig. 2 for some typical examples. When graphene is chemisorbed (on $\mathrm{Co}, \mathrm{Ni}$, and $\mathrm{Pd}$ ) the graphene bands are strongly perturbed and acquire a mixed graphene-metal character. In particular, the characteristic conical points at $\mathrm{K}$ are destroyed; see the bottom panels of Fig. 2 for the majority and minority spin bands of graphene on $\mathrm{Co}$. When the interaction is weaker $(\mathrm{Al}, \mathrm{Cu}, \mathrm{Ag}, \mathrm{Au}, \mathrm{Pt}$ ), the graphene bands, including their conical points at $\mathrm{K}$, can still be clearly identified; see the upper panels of Fig. 2. However, whereas in freestanding graphene the Fermi level coincides with the conical point, adsorption generally shifts the Fermi level. A shift upwards (downwards) means that electrons (holes) are donated by the metal substrate to graphene which becomes $n$-type ( $p$-type) doped.

For metal-graphene equilibrium separations, graphene is doped $n$-type on $\mathrm{Al}, \mathrm{Ag}$, and $\mathrm{Cu}$, and $p$-type on $\mathrm{Au}$ and $\mathrm{Pt}$; the corresponding Fermi level shifts are plotted in Fig. 3. Because the work functions of graphene, $W_{\mathrm{G}}$, and of most metal surfaces, $W_{\mathrm{M}}$, differ, as soon as graphene interacts
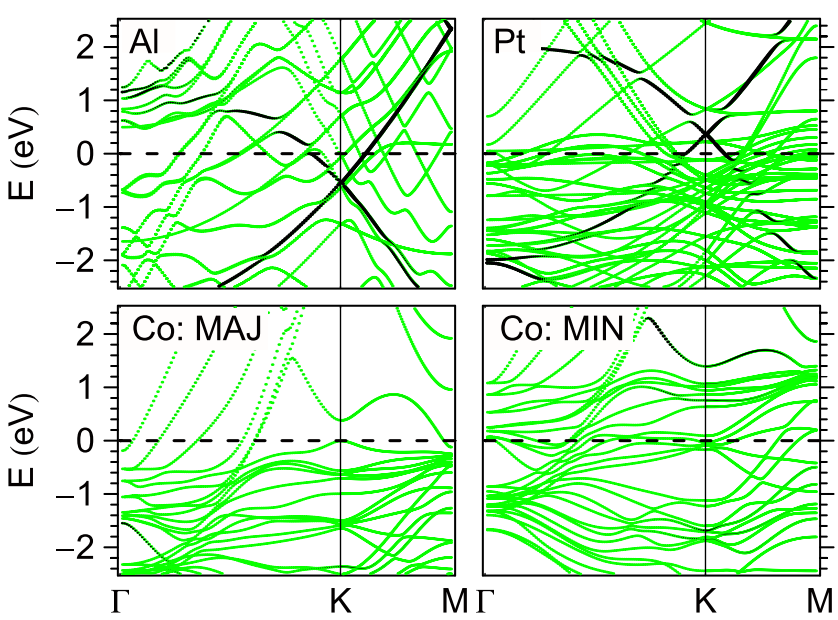

FIG. 2 (color online). Band structures of graphene absorbed upon Al, Pt, and Co (111) substrates. The bottom left and right panels correspond, respectively, to majority and minority spin band structures. The Fermi level is at zero energy. The amount of carbon $p_{z}$ character is indicated by the blackness of the bands. The conical point corresponds to the crossing of predominantly $p_{z}$ bands at $\mathrm{K}$. Note that on doubling the lattice vectors (for $\mathrm{Al}$ and $\mathrm{Pt}$ ), the $\mathrm{K}$ point is folded down onto the $\mathrm{K}$ point of the smaller Brillouin zone.

with a metal, electrons are transferred from one to the other to equilibrate the Fermi levels. A schematic representation is shown in Fig. 4 for the case of electron transfer from graphene to the metal. To a good approximation, the graphene DOS is described by $D(E)=D_{0}|E|$, with $D_{0}=$ 0.09 per $\left(\mathrm{eV}^{2}\right.$ unit cell) for $E$ within $1 \mathrm{eV}$ of the conical points. Since this DOS is much lower than that of the metal, equilibrium is effectively achieved by moving the Fermi level in graphene and even a small electron transfer will shift the Fermi level significantly. A transfer of 0.01 electrons would lower the Fermi level by $0.47 \mathrm{eV}$.

This electron transfer results in the formation of an interface dipole layer and an accompanying potential step $\Delta V$. We can use the plane-averaged electron densities $n(z)$ to visualize the electron redistribution $\Delta n(z)=n_{\mathrm{M} \mid \mathrm{G}}(z)-$ $n_{\mathrm{M}}(z)-n_{\mathrm{G}}(z)$ upon formation of the interface. As shown in Fig. 4, $\Delta n(z)$ is localized at the interface. The sign and size of the interface dipole are consistent with the changes

TABLE I. Calculated equilibrium separation $d_{\mathrm{eq}}$ of a graphene sheet from various metal (111) surfaces. The binding energy $\Delta E$ is the energy (per carbon atom) required to remove the graphene sheet from the metal surface. $W_{\mathrm{M}}$ and $W$ are, respectively, the work functions calculated for the clean metal surfaces, and for freestanding and adsorbed graphene.

\begin{tabular}{|c|c|c|c|c|c|c|c|c|c|}
\hline & $\mathrm{Gr}$ & $\mathrm{Ni}$ & $\mathrm{Co}$ & $\mathrm{Pd}$ & $\mathrm{Al}$ & $\mathrm{Ag}$ & $\mathrm{Cu}$ & $\mathrm{Au}$ & $\mathrm{Pt}$ \\
\hline$d_{\mathrm{eq}}(\AA)$ & & 2.05 & 2.05 & 2.30 & 3.41 & 3.33 & 3.26 & 3.31 & 3.30 \\
\hline$\Delta E(\mathrm{eV})$ & & 0.125 & 0.160 & 0.084 & 0.027 & 0.043 & 0.033 & 0.030 & 0.038 \\
\hline$W_{\mathrm{M}}(\mathrm{eV})$ & & 5.47 & 5.44 & 5.67 & 4.22 & 4.92 & 5.22 & 5.54 & 6.13 \\
\hline$W(\mathrm{eV})$ & 4.48 & 3.66 & 3.78 & 4.03 & 4.04 & 4.24 & 4.40 & 4.74 & 4.87 \\
\hline$W_{\text {expt }}(\mathrm{eV})$ & $4.6^{\mathrm{a}}$ & $3.9^{\mathrm{a}}$ & & $4.3^{\mathrm{a}}$ & & & & & $4.8^{\mathrm{a}}$ \\
\hline
\end{tabular}

${ }^{\mathrm{a}}$ Reference [8] 


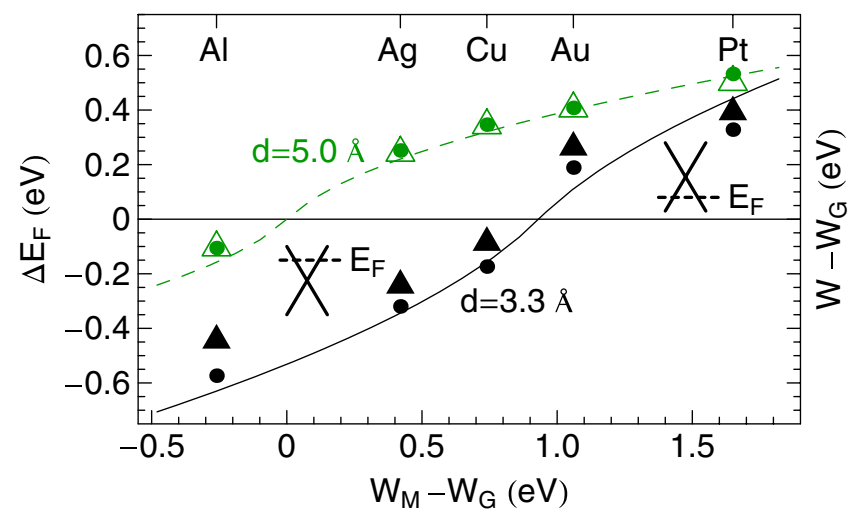

FIG. 3 (color online). Calculated Fermi energy shift with respect to the conical point, $\Delta E_{\mathrm{F}}$ (dots), and change in the work function $W-W_{\mathrm{G}}$ (triangles) as a function of $W_{\mathrm{M}}-W_{\mathrm{G}}$, the difference between the clean metal and graphene work functions. The lower (black) and the upper [gray (green)] results are for the equilibrium $(\sim 3.3 \AA)$ and a larger $(5.0 \AA)$ separation of graphene and the metal surfaces, respectively. The solid line and the dashed line follow from the model of Eq. (1) with $\Delta_{\mathrm{c}}=$ 0 for $d=5.0 \AA$. The insets illustrate the position of the Fermi level with respect to the conical point.

of the metal work function upon adsorption of graphene; see Table I.

Naively one would assume that graphene is doped with electrons if $W_{\mathrm{G}}>W_{\mathrm{M}}$ and doped with holes if $W_{\mathrm{G}}<W_{\mathrm{M}}$. The crossover point from $n$ - to $p$-type doping would then be at $W_{\mathrm{M}}=W_{\mathrm{G}}$. The results obtained at the equilibrium separations of the graphene sheet and the metal surfaces $(d \sim 3.3 \AA$; see Fig. 3) show that this is clearly not the case. Instead, the crossover point lies at $W_{\mathrm{M}}-W_{\mathrm{G}}=$ $0.9 \mathrm{eV}$. Only when the graphene-metal separation is increased significantly does the crossover point decrease to its expected value, as illustrated by the upper curve for $d=$ 5.0 A in Fig. 3. This clearly demonstrates that the charge redistribution at the graphene-metal interface is not only
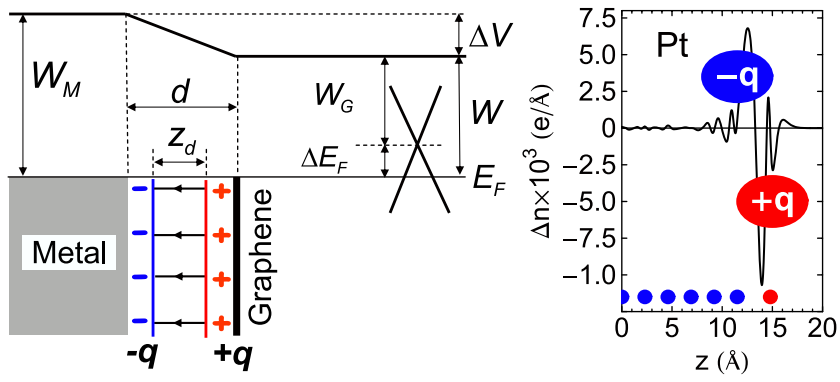

FIG. 4 (color online). Left: Schematic illustration of the parameters used in modeling the interface dipole and potential step formation at the graphene-metal interface. Right: Plane-averaged difference electron density $\Delta n(z)=n_{\mathrm{M} \mid \mathrm{G}}(z)-n_{\mathrm{M}}(z)-n_{\mathrm{G}}(z)$ showing the charge displacement upon formation of the graphene-Pt(111) interface. the result of an electron transfer between the metal and the graphene levels. There is also a contribution from a metalgraphene chemical interaction. Such an interaction, which has a significant repulsive contribution, has been found to play an important role in describing dipole formation when closed shell atoms and molecules are adsorbed on metal surfaces $[21,22]$.

The dependence of this interaction on the metalgraphene separation $d$ is mapped out in Fig. 5 in terms of the dependence of the Fermi level shift $\Delta E_{\mathrm{F}}$ on $d$. We use the parameters shown in Fig. 4 to construct a simple and general model with which to understand these results. The work function of the graphene-covered metal is given by $W(d)=W_{\mathrm{M}}-\Delta V(d)$ where $\Delta V$ is the potential change generated by the metal-graphene interaction. The Fermi level shift in graphene is modeled as $\Delta E_{\mathrm{F}}(d)=W(d)-$ $W_{\mathrm{G}}$. The key element is modeling the potential step $\Delta V=$ $\Delta_{\text {tr }}(d)+\Delta_{\mathrm{c}}(d)$ in terms of a "noninteracting" charge transfer contribution $\Delta_{\text {tr }}$ driven by the difference in work functions and a contribution $\Delta_{\mathrm{c}}$ resulting from the metalgraphene chemical interaction.

The charge transfer contribution is modeled by a plane capacitor model as indicated in Fig. 4. $\Delta_{\text {tr }}(d)=\alpha N(d) z_{d}$ where $\alpha=e^{2} / \varepsilon_{0} A=34.93 \mathrm{eV} / \AA$ with $A=5.18 \AA^{2}$ the area of the graphene unit cell and $N(d)$ is the number of electrons (per unit cell) transferred from graphene to the metal (becoming negative if electrons are transferred from the metal to graphene). $z_{d}$ is the effective distance between the charge sheets on graphene and the metal. $z_{d}<d$ as most of the charge is located between the graphene layer and the metal surface as illustrated in Fig. 4. We model it as $z_{d}=d-d_{0}$ with $d_{0}$ a constant.

Integrating the (linear) density of states of graphene yields a simple relation between $N(d)$ and $\Delta E_{\mathrm{F}}(d): N=$ $\pm D_{0} \Delta E_{F}^{2} / 2$. Using the relations introduced in the previous two paragraphs we can then express $\Delta E_{\mathrm{F}}(d)$ as

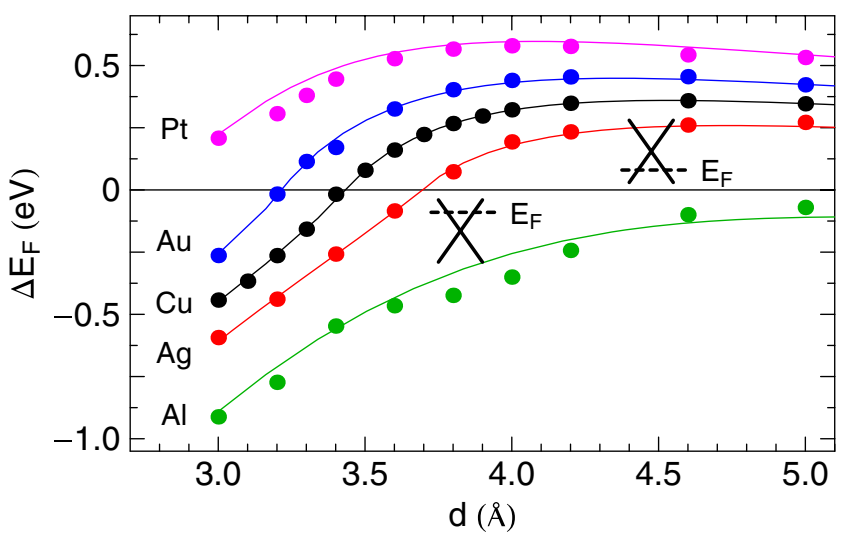

FIG. 5 (color online). Fermi level shifts $\Delta E_{\mathrm{F}}(d)$ as a function of the graphene-metal surface distance. The dots give the calculated DFT results, the solid lines give the results obtained from the model, Eq. (1) [23]. 


$$
\Delta E_{\mathrm{F}}(d)= \pm \frac{\sqrt{1+2 \alpha} \overline{D_{0}\left(d-d_{0}\right)\left|W_{\mathrm{M}}-W_{\mathrm{G}}-\bar{\Delta}_{\mathrm{c}}(\bar{d})\right|}-1}{\alpha D_{0}\left(d-d_{0}\right)},
$$

where the sign of $\Delta E_{\mathrm{F}}$ is given by the sign of $W_{\mathrm{M}}-W_{\mathrm{G}}-$ $\Delta_{\mathrm{c}}$. The parameters $d_{0}$ and $\Delta_{\mathrm{c}}(d)$ turn out to depend only very weakly on the metal substrate. If we fit these quantities to the DFT results for one metal substrate, we can use them as universal parameters to predict the Fermi level shifts in graphene for all metal substrates. We use the DFT results obtained for graphene on $\mathrm{Cu}(111)$ to fix $d_{0}$ and $\Delta_{\mathrm{c}}(d)$; see Ref. [23]. Only the work function of the clean metal surface, $W_{\mathrm{M}}$, and that of freestanding graphene, $W_{\mathrm{G}}$, are then needed to calculate the Fermi level shift. The accuracy of the model represented by Eq. (1) is demonstrated in Figs. 3 and 5. From $\Delta E_{\mathrm{F}}$ one can immediately obtain the work function $W$ of the metal-graphene system, as well as the sign and concentration of the charge carriers in graphene, $N$.

The critical metal work function $W_{\mathrm{M}}=W_{0}$, where the Fermi level is at the conical points of graphene, can be obtained from Eq. (1) for $\Delta E_{\mathrm{F}}(d)=0$. It gives $W_{0}(d)=$ $W_{\mathrm{G}}+\Delta_{\mathrm{c}}(d)$. The contribution of the chemical interaction term $\Delta_{\mathrm{c}}$ depends strongly on the distance $d$ between graphene and the metal surface. At a large distance $d \gtrsim 4.2 \AA$, $\Delta_{\mathrm{c}} \ll 1 \mathrm{eV}$ and $W_{0}(d) \approx W_{\mathrm{G}}=4.5 \mathrm{eV}$, whereas at the equilibrium separation $d_{\mathrm{eq}}=3.3 \AA, \Delta_{\mathrm{c}} \approx 0.9 \mathrm{eV}$ and $W_{0}(d) \approx 5.4 \mathrm{eV}$. This agrees with the DFT results shown in Fig. 3. The chemical interaction thus leads to a sizable potential step at the equilibrium separation, which is downwards from metal to graphene as indicated in Fig. 4. The sign of this step and its insensitivity to the metal substrate are consistent with its interpretation in terms of an exchange repulsion between the electrons on graphene and the metal substrate [22].

In conclusion, we have used DFT calculations to study the doping of graphene induced by adsorption on metal surfaces and developed a simple model that takes into account the electron transfer between the metal and graphene levels driven by the work function difference, as well as the chemical interaction between graphene and the metal. The model extends the applicability of the detailed DFT results to the more complex systems encountered in practical devices and suggests combinations of metal (strips) to be used to realize $p-n$ junctions [24].

We thank Paul Rusu for helpful discussions. This work was financially supported by "NanoNed" (a program of the Dutch Ministry of Economic Affairs) and by the "Nederlandse Organisatie voor Wetenschappelijk Onderzoek (NWO)" via "Chemische Wetenschappen (CW)" and "Stichting voor Fundamenteel Onderzoek der Materie (FOM)." Computer facilities were granted by "Stichting Nationale Computerfaciliteiten (NCF)."
[1] K. S. Novoselov et al., Science 306, 666 (2004).

[2] K. S. Novoselov et al., Nature (London) 438, 197 (2005).

[3] N.H. Shon and T. Ando, J. Phys. Soc. Jpn. 67, 2421 (1998).

[4] T. Ando et al., J. Phys. Soc. Jpn. 71, 1318 (2002).

[5] V.P. Gusynin and S. G. Sharapov, Phys. Rev. Lett. 95, 146801 (2005).

[6] M. I. Katsnelson et al., Nature Phys. 2, 620 (2006).

[7] J. van den Brink, Nature Nanotechnology 2, 199 (2007).

[8] C. Oshima and A. Nagashima, J. Phys. Condens. Matter 9, 1 (1997).

[9] Yu. S. Dedkov et al., Phys. Rev. B 64, 035405 (2001).

[10] G. Bertoni et al., Phys. Rev. B 71, 075402 (2005).

[11] A.T. N'Diaye et al., Phys. Rev. Lett. 97, 215501 (2006).

[12] V. M. Karpan et al., Phys. Rev. Lett. 99, 176602 (2007).

[13] G. Giovannetti et al., Phys. Rev. B 76, 073103 (2007).

[14] S. Marchini et al., Phys. Rev. B 76, 075429 (2007).

[15] B. Uchoa et al., Phys. Rev. B 77, 035420 (2008).

[16] H. Schomerus, Phys. Rev. B 76, 045433 (2007).

[17] Ya. M. Blanter and I. Martin, Phys. Rev. B 76, 155433 (2007).

[18] We use a plane wave basis set with a kinetic energy cutoff of $400 \mathrm{eV}$ in the projector augmented-wave formalism, at the level of the local spin density approximation, as implemented in VASP [G. Kresse and J. Furthmuller, Phys. Rev. B 54, 11169 (1996); Comput. Mater. Sci. 6, 15 (1996)]. The supercell contains a slab of six layers of metal atoms with a graphene sheet adsorbed on one side and a vacuum region of $\sim 12 \AA$. A dipole correction is used [J. Neugebauer and M. Scheffler, Phys. Rev. B 46, 16067 (1992)]. We apply $36 \times 36$ and $24 \times 24$ k-point grids to sample the Brillouin zone of the small and large cells in Fig. 1, respectively, and use the tetrahedron method for Brillouin zone integrations that include the $\Gamma, \mathrm{K}$, and $\mathrm{M}$ special points. Total energies are converged to $10^{-7} \mathrm{eV}$.

[19] Y. Gamo et al., Surf. Sci. 374, 61 (1997).

[20] Yue Qi et al., Surf. Sci. 581, 155 (2005).

[21] J.L.F. Silva et al., Phys. Rev. Lett. 90, 066104 (2003).

[22] P. C. Rusu, Ph.D. thesis, University of Twente, 2007; see http://purl.org/utwente/58034.

[23] We obtain $\Delta_{\mathrm{c}}(d)$ by least-squares fitting Eq. (1) to the DFT results for $\Delta E_{\mathrm{F}}(d)$ for $\mathrm{Cu}(111)$ with $d_{0}=2.4 \AA$. This value of $d_{0}$ provides the best fit of $\Delta E_{\mathrm{F}}(d)$ for other metal surfaces. At large $d$ the chemical interaction term $\Delta_{\mathrm{c}}(d)$ should vanish. Therefore, parametrizing $\quad \Delta_{\mathrm{c}}(d)=e^{-\kappa d}\left(a_{0}+a_{1} d+a_{2} d^{2}\right)$ gives $\kappa=$ $1.6443 \AA^{-1}, a_{0}=-2048.56 \mathrm{eV}, a_{1}=1363.87 \mathrm{eV} / \AA$, $a_{2}=-205.737 \mathrm{eV} / \AA^{2}$, where $d \gtrsim 3.0 \AA$. The general applicability of this equation to all metal substrates can be explained from the weak metal-graphene interaction. The charge redistribution is then dominated by exchange repulsion and is almost independent of the metal species [22].

[24] V. V. Cheianov and V.I. Fal'ko, Phys. Rev. B 74, 041403 (2006); C.-H. Park et al., Nature Phys. 4, 213 (2008). 